\title{
Convex Properties of Center-of-Mass Trajectories for Locomotion Based on Divergent Component of Motion
}

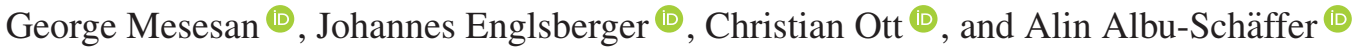

\begin{abstract}
This letter presents an in-depth analysis of the convex properties of center-of-mass $(\mathrm{CoM})$ trajectories for legged robot locomotion based on the concept of Divergent Component of Motion (DCM). In particular, we show that the union of all possible trajectories forms a bounded convex set under appropriate boundary conditions. Additionally, we describe in detail our approach of generating closed-form CoM trajectories through piecewise interpolation over a sequence of waypoints and show how to compute the CoM trajectory efficiently through equations given in a matrix form. Applying the convex properties to our trajectory-generation approach, we present an algorithm for computing convex overapproximations of the CoM waypoints. Finally, we provide an example of usage in placing waypoints that lead to feasible CoM trajectories with respect to kinematic and dynamic constraints. The approach is validated with a multi-contact scenario in simulation with the humanoid robot TORO.
\end{abstract}

Index Terms-Computational geometry, divergent component of motion, humanoid and bipedal locomotion, legged robots, motion and path planning.

\section{INTRODUCTION}

L OCOMOTION of legged robots is a challenging problem due to its hybrid dynamics (discrete contact sequencing and continuous whole-body motion), and the constraints on the direction and amplitude of the contact forces. While attempts have been made to plan the motion directly in the robot joint space [1], [2], a more promising approach has been to focus on the motion of the center of mass $(\mathrm{CoM})$ with respect to the contact points, which has been shown to be consistent with legged locomotion of biological systems [3].

Following the introduction of the Zero-Moment Point (ZMP) [4] and Linear Inverted Pendulum (LIP) [5], several methods of generating CoM trajectories based on these two concepts were presented in the literature. Harada et al. [6] use a

Manuscript received February 24, 2018; accepted June 19, 2018. Date of publication July 5, 2018; date of current version August 2, 2018. This letter was recommended for publication by Associate Editor Z. Li and Editor N. Tsagarakis upon evaluation of the reviewers' comments. This work was supported in part by the European Commission under Grant H2020-ICT-645097 COMANOID. (Corresponding author: George Mesesan.)

The authors are with the Institute of Robotics and Mechatronics, German Aerospace Center (DLR), 82234 Wessling, Germany (e-mail: george.mesesan@dlr.de; johannes.englsberger@dlr.de; christian.ott@dlr.de; alin.albu-schaeffer@dlr.de).

Digital Object Identifier 10.1109/LRA.2018.2853557 piecewise spline interpolation for the ZMP, and give an analytical solution for the CoM trajectory, where both the initial and the final position of the CoM are specified. Kajita et al. [7] generate the CoM trajectory as piecewise cubic polynomials, and minimize the CoM jerk for a predefined ZMP trajectory using Model Predictive Control (MPC). A closed-form solution for the CoM trajectory without using the CoM jerk is presented by Tedrake et al. in [8]. Extending the ZMP to multiple noncoplanar contacts led to the introduction of the Contact Wrench Cone (CWC) [9], later simplified and applied to multi-contact motion by Caron et al. [10]. The CWC was used for CoM trajectory generation via convex optimization by Dai et al. [11], and for computing convex CoM regions for static [12] and dynamic [13] stability.

Recently, the concepts of three-dimensional Divergent Component of Motion (DCM) and Virtual Repellent Point (VRP) were introduced in [14], decomposing the second-order CoM dynamics into two first-order linear dynamics, with the CoM converging to the DCM (stable dynamics), and the DCM diverging away from the VRP (unstable dynamics) [14], [15]. Based on this formulation, closed-form DCM and CoM trajectories can be generated using a piecewise interpolation of the VRP trajectory over a sequence of waypoints. This highly compact motion representation is a natural way of handling the hybrid dynamics discussed above, with the discrete contact sequencing being mapped onto the VRP waypoints. This approach has been successfully validated in our previous work: for dynamic walking in [14], and dynamic multi-contact locomotion in [16]. Furthermore, the closed-form trajectories enable the use of efficient search algorithms for planning, such as the binary search approach for finding the contact transition timing in [16].

The main contributions of this work are: (i) we formally prove convex properties of the DCM and CoM trajectories for arbitrary VRP trajectories; (ii) for piecewise interpolation trajectories, we introduce an algorithm for computing overapproximating convex hulls of the CoM waypoints; (iii) we present optimal VRP waypoint placement using the CoM waypoint convex hulls. While we give only one example of usage in this letter, we anticipate various applications for the convex properties of DCM-based trajectories in locomotion planning and control. For example, the CoM region of dynamic stability introduced in [13] can be combined with the CoM trajectory convex hull to produce a region of dynamically feasible and reachable $\mathrm{CoM}$ points. 
The rest of the paper is structured as follows: in Section II we prove the convex properties of the DCM-based trajectories, while in Section III, we give a detailed treatment of CoM trajectory generation based on VRP piecewise interpolation, extending our work from [17] with CoM waypoint and trajectory computations in matrix form. Based on the results from Sections II and III, we introduce an algorithm for computing overapproximating convex hulls for the region of reachable CoM positions in Section IV. We give an example of usage by extending our multi-contact motion planner [16] with optimal VRP waypoint placement in Section V, followed by the implementation details and simulation results in Section VI. Section VII concludes the paper.

\section{Convex Properties of DCM-BASEd TRAJECTORIES}

The three-dimensional Divergent Component of Motion (DCM) $\boldsymbol{\xi}$ was defined in [14] as a linear combination of the CoM position $\boldsymbol{x}$ and velocity $\dot{\boldsymbol{x}}$ :

$$
\boldsymbol{\xi}=\boldsymbol{x}+b \dot{\boldsymbol{x}},
$$

where $b$ is a time constant defined as $b=\sqrt{\frac{\Delta z}{g}}$. Here, $\Delta z$ represents the average CoM height above the ground surface, and $g$ denotes the gravitational constant.

The Virtual Repellent Point (VRP) $\boldsymbol{v}$ was introduced in [14] as a linear combination of the CoM position $\boldsymbol{x}$ and acceleration $\ddot{x}$ :

$$
\boldsymbol{v}=\boldsymbol{x}-b^{2} \ddot{\boldsymbol{x}} .
$$

The VRP encodes the effects of the total force $f$ (i.e., gravity and all external forces) acting on the CoM, as the CoM acceleration is proportional to the total force (Newton's 2nd law), $\ddot{\boldsymbol{x}}=\frac{f}{m}$, with $m$ being the mass of the robot. From (1) and (2) we find the relation between DCM and VRP to be

$$
\boldsymbol{v}=\boldsymbol{\xi}-b \dot{\boldsymbol{\xi}} \text {. }
$$

\section{A. Convexity Proof}

We prove the convexity of the DCM and CoM trajectories using the concept of reachable set [18]. For the CoM dynamics

$$
\dot{\boldsymbol{x}}=-\frac{1}{b} \boldsymbol{x}+\frac{1}{b} \boldsymbol{\xi},
$$

obtained by reordering (1), we denote the locus of the CoM position at time $t$ as

$$
\mathcal{X}(t)=\left\{\boldsymbol{x}(t) \mid \boldsymbol{x}_{0} \in \mathcal{H}_{x_{0}}, \boldsymbol{\xi} \in \mathcal{H}_{\xi}\right\},
$$

where

$$
\boldsymbol{x}(t)=e^{-\frac{t}{b}} \boldsymbol{x}_{0}+\frac{1}{b} \int_{0}^{t} e^{-\frac{t-\tau}{b}} \boldsymbol{\xi}(\tau) d \tau,
$$

with $\boldsymbol{x}_{0}$ being the initial CoM position. We consider the sets $\mathcal{H}_{x_{0}}$ and $\mathcal{H}_{\xi}$ to be bounded convex polyhedra, such that we can enumerate their vertices. Let $\boldsymbol{x}_{0}^{j}$ be an arbitrary vertex of $\mathcal{H}_{x_{0}}$, and $\boldsymbol{\xi}^{k}$ an arbitrary vertex of $\mathcal{H}_{\xi}$. For the initial CoM position $\boldsymbol{x}_{0}^{j}$ and constant DCM $\boldsymbol{\xi}=\boldsymbol{\xi}^{k}$, we find from (6) the CoM position at time $t$ to be

$$
\boldsymbol{x}(t)=e^{-\frac{t}{b}} \boldsymbol{x}_{0}^{j}+\left(1-e^{-\frac{t}{b}}\right) \boldsymbol{\xi}^{k} .
$$

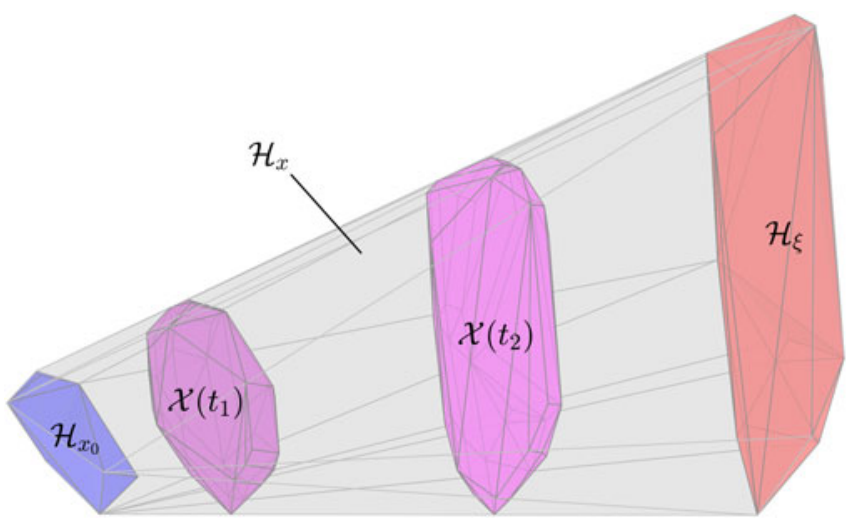

Fig. 1. CoM convex hull example.

Using the convexity property of $\mathcal{X}(t)$ [18], we obtain

$$
\mathcal{X}(t)=e^{-\frac{t}{b}} \mathcal{H}_{x_{0}} \oplus\left(1-e^{-\frac{t}{b}}\right) \mathcal{H}_{\xi}
$$

The set of all possible CoM positions, i.e., the union of all possible CoM trajectories, can be written as the reachable set $\mathcal{H}_{x}=\cup_{t \geqslant 0} \mathcal{X}(t)$, which combined with (8) leads to

$$
\mathcal{H}_{x}=\operatorname{CoNv}\left(\mathcal{H}_{x_{0}} \cup \mathcal{H}_{\xi}\right),
$$

where the operator $\operatorname{CONV}(\cdot)$ returns the convex hull of a set of points, and we used the property $0 \leqslant e^{-\frac{t}{b}} \leqslant 1, \forall t \geqslant 0$. Figure 1 shows an example of the shape of $\mathcal{H}_{x}$ given arbitrary sets $\mathcal{H}_{x_{0}}$ and $\mathcal{H}_{\xi}$. Also shown are two instances of the set $\mathcal{X}(t)$ at $t_{1}$ and $t_{2}$ with the relation $0<t_{1}<t_{2}$.

For the DCM trajectory, we consider the negative-time DCM dynamics

$$
\dot{\boldsymbol{\xi}}_{r}=-\frac{1}{b} \boldsymbol{\xi}+\frac{1}{b} \boldsymbol{v}
$$

obtained by reordering (3), and reversing the sign of the time variable $t$, with $\dot{\boldsymbol{\xi}}_{r}=-\dot{\boldsymbol{\xi}}$ being the negative-time derivative of the DCM. As shown in [19], the reachable set of the negativetime system is identical with the recoverable set [19] of the positive-time system, this being, in our case, the union of all DCM trajectories ending in a given target set $\mathcal{H}_{\xi_{T}}$. Due to the similarity between (10) and (4), we can use the same reasoning as above, considering the set of target DCM positions $\mathcal{H}_{\xi_{T}}$ and the set of VRP positions $\mathcal{H}_{v}$ to be bounded convex polyhedra. We obtain thus

$$
\mathcal{H}_{\xi}=\operatorname{CoNV}\left(\mathcal{H}_{\xi_{T}} \cup \mathcal{H}_{v}\right)
$$

\section{TRAJECTORY GENERATION}

In this work, we generate CoM trajectories by splitting the motion into a sequence of $n_{\varphi}$ transition phases. As boundary conditions for the complete motion we choose a DCM target point $\boldsymbol{\xi}_{f}$ and a CoM start point $\boldsymbol{x}_{s}$. For each transition phase $\varphi \in\left\{1, \ldots, n_{\varphi}\right\}$, we specify the VRP trajectory $\boldsymbol{v}_{\varphi}(t)$ and solve the differential equations (1) and (3) with appropriate boundary conditions to obtain the DCM trajectory $\boldsymbol{\xi}_{\varphi}(t)$ and CoM trajectory $\boldsymbol{x}_{\varphi}(t)$. We denote by $\boldsymbol{v}_{\varphi, 0}, \boldsymbol{\xi}_{\varphi, 0}$, and $\boldsymbol{x}_{\varphi, 0}$ the start points, and by $\boldsymbol{v}_{\varphi, T}, \boldsymbol{\xi}_{\varphi, T}$, and $\boldsymbol{x}_{\varphi, T}$ the end points of the VRP, DCM, and CoM transition phase trajectories, respectively. The continuity of the complete trajectories is ensured by linking 


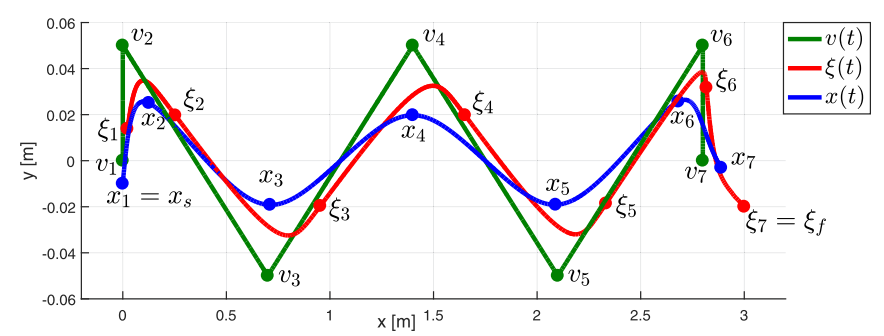

Fig. 2. Generated trajectory example ( $x y$-view).

the start points of a transition phase with the end points of the previous transition phase, i.e., the following equalities hold for all transition phases: $\boldsymbol{v}_{\varphi, 0}=\boldsymbol{v}_{\varphi-1, T}, \boldsymbol{\xi}_{\varphi, 0}=\boldsymbol{\xi}_{\varphi-1, T}$, and $\boldsymbol{x}_{\varphi, 0}=\boldsymbol{x}_{\varphi-1, T}$.

Equivalently, the complete VRP trajectory $\boldsymbol{v}(t)$ can be described as a piecewise interpolation function over a sequence of $n=n_{\varphi}+1$ VRP waypoints $\left(\boldsymbol{v}_{i}\right)_{i=1}^{n}$, with analogous interpretations for the DCM trajectory $\boldsymbol{\xi}(t)$ with DCM waypoints $\left(\boldsymbol{\xi}_{i}\right)_{i=1}^{n}$, and for the CoM trajectory $\boldsymbol{x}(t)$ with CoM waypoints $\left(\boldsymbol{x}_{i}\right)_{i=1}^{n}$. For example, for the first transition phase $(\varphi=1), \boldsymbol{v}_{1}$, $\boldsymbol{\xi}_{1}$, and $\boldsymbol{x}_{1}$ are the start points, and $\boldsymbol{v}_{2}, \boldsymbol{\xi}_{2}$, and $\boldsymbol{x}_{2}$ are the end points of the VRP, DCM, and CoM transition phase trajectories, respectively. Figure 2 shows an example of a sequence of 7 waypoints, displaying generated trajectories and computed waypoints for VRP, DCM and CoM, with duration $T_{\varphi}=0.75 \mathrm{~s}$ for all transition phases.

In this section, we focus first on a single transition phase, deriving closed-form DCM and CoM trajectories, and discussing their respective convex properties. Subsequently, we present an efficient way of computing the DCM and CoM waypoints, $\left(\boldsymbol{\xi}_{i}\right)_{i=1}^{n}$ and $\left(\boldsymbol{x}_{i}\right)_{i=1}^{n}$, respectively, in terms of $\left(\boldsymbol{v}_{i}\right)_{i=1}^{n}, \boldsymbol{\xi}_{f}$, and $\boldsymbol{x}_{s}$.

\section{A. Trajectories During a Single Transition Phase}

VRP Trajectory [17]: We define the VRP trajectory $\boldsymbol{v}_{\varphi}(t)$ as a spatial linear interpolation between the VRP start point $\boldsymbol{v}_{\varphi, 0}$ and the corresponding VRP end point $\boldsymbol{v}_{\varphi, T}$ with the following general form:

$$
\boldsymbol{v}_{\varphi}(t)=\left(1-f_{\varphi}(t)\right) \boldsymbol{v}_{\varphi, 0}+f_{\varphi}(t) \boldsymbol{v}_{\varphi, T},
$$

where $t \in\left[0, T_{\varphi}\right]$ is the local time of the transition phase, with $T_{\varphi}$ being a constant, strictly positive value, denoting the phase duration. The temporal interpolation function $f_{\varphi}(t)$ is a polynomial of degree $p$ with the following properties: $f_{\varphi}(0)=0, f_{\varphi}\left(T_{\varphi}\right)=1$, and $0 \leqslant f_{\varphi}(t) \leqslant 1, \forall t \in\left[0, T_{\varphi}\right]$. For example, a first-order polynomial with the required properties is $f_{\varphi}(t)=t / T_{\varphi}$, while a third-order polynomial $(p=3)$ is $f_{\varphi}(t)=3\left(t / T_{\varphi}\right)^{2}-2\left(t / T_{\varphi}\right)^{3}$ (more examples with corresponding boundary conditions are given in [17]).

The set of VRP positions during the transition phase $\varphi$ is, by design, the bounded convex set

$$
\mathcal{H}_{\varphi, v}=\operatorname{CoNV}\left(\left\{\boldsymbol{v}_{\varphi, 0}, \boldsymbol{v}_{\varphi, T}\right\}\right) .
$$

DCM Trajectory [17]: We derive the DCM trajectory by replacing (12) in (3) and solving the resulting linear differential equation using the DCM end point $\boldsymbol{\xi}_{\varphi}\left(T_{\varphi}\right)=\boldsymbol{\xi}_{\varphi, T}$ as boundary condition, to obtain a closed-form solution for the DCM

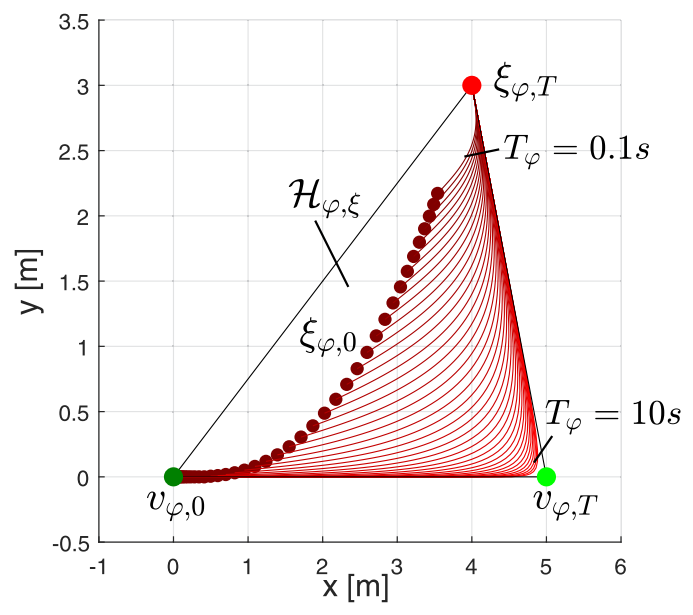

Fig. 3. Example of DCM trajectories (red curves).

trajectory:

$$
\begin{aligned}
\boldsymbol{\xi}_{\varphi}(t)= & \underbrace{\left(1-\sigma_{\varphi}(t)-e^{\frac{t-T_{\varphi}}{b}}\left(1-\sigma_{\varphi, T}\right)\right)}_{\alpha_{\varphi, \xi}(t)} \boldsymbol{v}_{\varphi, 0} \\
& +\underbrace{\left(\sigma_{\varphi}(t)-e^{\frac{t-T \varphi}{b}} \sigma_{\varphi, T}\right)}_{\beta_{\varphi, \xi}(t)} \boldsymbol{v}_{\varphi, T}+\underbrace{e^{\frac{t-T \varphi}{b}}}_{\gamma_{\varphi, \xi}(t)} \boldsymbol{\xi}_{\varphi, T},
\end{aligned}
$$

where

$$
\sigma_{\varphi}(t)=\sum_{k=0}^{p}\left(b^{k} f_{\varphi}^{(k)}(t)\right)
$$

and $\sigma_{\varphi, T}:=\sigma_{\varphi}\left(T_{\varphi}\right)$. Here, the notation $f_{\varphi}(t)$ denotes the $k$-th time derivative of $f_{\varphi}(t)$. Applying (11) with (13) and $\mathcal{H}_{\varphi, \xi_{T}}=\left\{\boldsymbol{\xi}_{\varphi, T}\right\}$, we obtain the convex hull of the DCM trajectories during the transition phase $\varphi$ :

$$
\mathcal{H}_{\varphi, \xi}=\operatorname{CONV}\left(\left\{\boldsymbol{v}_{\varphi, 0}, \boldsymbol{v}_{\varphi, T}, \boldsymbol{\xi}_{\varphi, T}\right\}\right),
$$

which shows that $\boldsymbol{\xi}_{\varphi}(t)$ is a convex combination of $\boldsymbol{v}_{\varphi, 0}, \boldsymbol{v}_{\varphi, T}$, and $\boldsymbol{\xi}_{\varphi, T}$, i.e., the coefficients $\alpha_{\varphi, \xi}(t), \beta_{\varphi, \xi}(t)$, and $\gamma_{\varphi, \xi}(t)$ are nonnegative and their sum equals $1, \forall t \in\left[0, T_{\varphi}\right]$. Figure 3 shows an example of DCM trajectories for a fifth order polynomial $f_{\varphi}(t)$ and different durations $T_{\varphi} \in[0.1,10] \mathrm{s}$.

CoM Trajectory: Similarly to the DCM trajectory, we derive the CoM trajectory by replacing (14) in (1) and solving the resulting linear differential equation using the CoM start point $\boldsymbol{x}_{\varphi}(0)=\boldsymbol{x}_{\varphi, 0}$ as boundary condition, to obtain a closed-form solution for the CoM trajectory:

$$
\begin{aligned}
\boldsymbol{x}_{\varphi}(t)= & \underbrace{\left(1-\rho_{\varphi}(t)-\frac{1-\rho_{\varphi, 0}}{e^{\frac{t}{b}}}-\frac{e^{\frac{t}{b}}-e^{-\frac{t}{b}}}{2 e^{\frac{T \varphi}{b}}}\left(1-\sigma_{\varphi, T}\right)\right)}_{\alpha_{\varphi, x}(t)} \boldsymbol{v}_{\varphi, 0} \\
& +\underbrace{\left(\rho_{\varphi}(t)-\frac{\rho_{\varphi, 0}}{e^{\frac{t}{b}}}-\frac{e^{\frac{t}{b}}-e^{-\frac{t}{b}}}{2 e^{\frac{T \varphi}{b}}} \sigma_{\varphi, T}\right)}_{\beta_{\varphi, x}(t)} \boldsymbol{v}_{\varphi, T} \\
& +\underbrace{\frac{e^{\frac{t}{b}}-e^{-\frac{t}{b}}}{2 e^{\frac{T \varphi}{b}}}}_{\gamma_{\varphi, x}(t)} \boldsymbol{\xi}_{\varphi, T}+\underbrace{e^{-\frac{t}{b}}}_{\delta_{\varphi, x}(t)} \boldsymbol{x}_{\varphi, 0},
\end{aligned}
$$




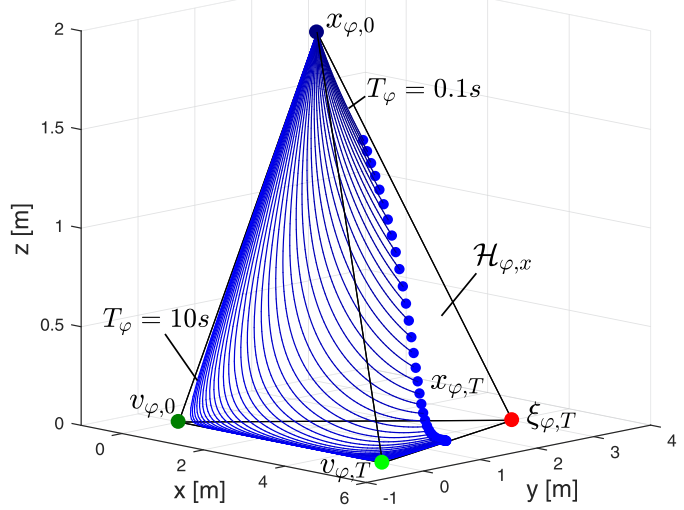

Fig. 4. Example of CoM trajectories (blue curves).

where

$$
\rho_{\varphi}(t)=\sum_{k=0}^{\lfloor p / 2\rfloor}\left(b^{2 k} \stackrel{(2 k)}{f}_{\varphi}(t)\right),
$$

and $\rho_{\varphi, 0}:=\rho_{\varphi}(0)$. Applying (9) with (16) and $\mathcal{H}_{\varphi, x_{0}}=\left\{\boldsymbol{x}_{\varphi, 0}\right\}$, we obtain the convex hull of the CoM trajectories during the transition phase $\varphi$ :

$$
\mathcal{H}_{\varphi, x}=\operatorname{CoNv}\left(\left\{\boldsymbol{v}_{\varphi, 0}, \boldsymbol{v}_{\varphi, T}, \boldsymbol{\xi}_{\varphi, T}, \boldsymbol{x}_{\varphi, 0}\right\}\right),
$$

which shows that $\boldsymbol{x}_{\varphi}(t)$ is a convex combination of $\boldsymbol{v}_{\varphi, 0}, \boldsymbol{v}_{\varphi, T}$, $\boldsymbol{\xi}_{\varphi, T}$, and $\boldsymbol{x}_{\varphi, 0}$, i.e., the coefficients $\alpha_{\varphi, x}(t), \beta_{\varphi, x}(t), \gamma_{\varphi, x}(t)$, and $\delta_{\varphi, x}(t)$ are nonnegative and their sum equals $1, \forall t \in\left[0, T_{\varphi}\right]$. Figure 4 shows an example of CoM trajectories for the same parameters as Fig. 3.

\section{B. Efficient Computation of the DCM and CoM Waypoints}

We collect the VRP waypoints $\boldsymbol{v}_{i}$ in a matrix $\boldsymbol{V}=\left[\boldsymbol{v}_{1} \cdots \boldsymbol{v}_{n}\right]^{T} \in \mathbb{R}^{n \times 3}$, the DCM waypoints $\boldsymbol{\xi}_{i}$ in a matrix $\boldsymbol{\Xi}=\left[\boldsymbol{\xi}_{1} \cdots \boldsymbol{\xi}_{n}\right]^{T}$, and the CoM waypoints $\boldsymbol{x}_{i}$ in $\boldsymbol{X}=\left[\boldsymbol{x}_{1} \cdots \boldsymbol{x}_{n}\right]^{T}$. Our goal is to obtain equations in matrix form for computing $\boldsymbol{\Xi}$ and $\boldsymbol{X}$ in terms of $\boldsymbol{V}$, the DCM target point $\boldsymbol{\xi}_{f}$, and the CoM start point $\boldsymbol{x}_{s}$, by using the equations (14) and (17). As these equations are expressed in terms of the transition phase start and end points $\left(\boldsymbol{v}_{\varphi, 0}, \boldsymbol{v}_{\varphi, T}\right.$, etc.), we introduce two selection matrices. $\boldsymbol{S}_{0}=\left[\begin{array}{ll}\boldsymbol{I}_{n_{\varphi} \times n_{\varphi}} & \mathbf{0}_{n_{\varphi} \times 1}\end{array}\right]$ selects the transition phase start points, while $\boldsymbol{S}_{T}=\left[\begin{array}{ll}\mathbf{0}_{n_{\varphi} \times 1} & \boldsymbol{I}_{n_{\varphi} \times n_{\varphi}}\end{array}\right]$ selects the transition phase end points from any waypoint matrix $(\boldsymbol{V}, \boldsymbol{\Xi}$, or $\boldsymbol{X})$. We denote by $\boldsymbol{V}_{0}=\boldsymbol{S}_{0} \boldsymbol{V} \in \mathbb{R}^{n_{\varphi} \times 3}$ the VRP start points, by $\boldsymbol{V}_{T}=\boldsymbol{S}_{T} \boldsymbol{V} \in \mathbb{R}^{n_{\varphi} \times 3}$ the VRP end points, and, analogously, we define $\boldsymbol{\Xi}_{0}, \boldsymbol{\Xi}_{T}, \boldsymbol{X}_{0}$, and $\boldsymbol{X}_{T}$, containing the DCM and CoM start and end points, respectively.

DCM Waypoints: We start by evaluating (14) for $t=0$ :

$$
\boldsymbol{\xi}_{\varphi, 0}=\alpha_{\varphi, \xi_{0}} \boldsymbol{v}_{\varphi, 0}+\beta_{\varphi, \xi_{0}} \boldsymbol{v}_{\varphi, T}+\gamma_{\varphi, \xi_{0}} \boldsymbol{\xi}_{\varphi, T},
$$

where $\alpha_{\varphi, \xi_{0}}:=\alpha_{\varphi, \xi}(0), \quad \beta_{\varphi, \xi_{0}}:=\beta_{\varphi, \xi}(0), \quad$ and $\quad \gamma_{\varphi, \xi_{0}}:=$ $\gamma_{\varphi, \xi}(0)$. We write (20) in matrix form for all $n_{\varphi}$ phases as

$$
\boldsymbol{\Xi}_{0}=\boldsymbol{A}_{\xi} \boldsymbol{V}_{0}+\boldsymbol{B}_{\xi} \boldsymbol{V}_{T}+\boldsymbol{\Gamma}_{\xi} \boldsymbol{\Xi}_{T},
$$

where $\boldsymbol{A}_{\xi}, \boldsymbol{B}_{\xi}$, and $\boldsymbol{\Gamma}_{\xi}$ are square, diagonal matrices containing the coefficients $\alpha_{\varphi, \xi_{0}}, \beta_{\varphi, \xi_{0}}$, and $\gamma_{\varphi, \xi_{0}}$, respectively. We use a terminal constraint for the DCM, $\boldsymbol{\xi}_{n}=\boldsymbol{\xi}_{f},{ }^{1}$ which can be expressed in terms of $\boldsymbol{\Xi}$ as

$$
\underbrace{\left[\begin{array}{cc}
\mathbf{0}_{n_{\varphi} \times n_{\varphi}} & \mathbf{0}_{n_{\varphi} \times 1} \\
\mathbf{0}_{1 \times n_{\varphi}} & 1
\end{array}\right]}_{\boldsymbol{S}_{n} \in \mathbb{R}^{n \times n}} \boldsymbol{\Xi}=\underbrace{\left[\begin{array}{c}
\mathbf{0}_{n_{\varphi} \times 1} \\
1
\end{array}\right]}_{\boldsymbol{s}_{f} \in \mathbb{R}^{n}} \boldsymbol{\xi}_{f}^{T} .
$$

Rewriting (21) in terms of $\boldsymbol{V}$ and $\boldsymbol{\Xi}$, multiplying it on the left with $\boldsymbol{S}_{0}^{T}$, adding (22), and grouping the $\boldsymbol{\Xi}$ terms on the left side of the equation yields

$$
\begin{aligned}
&\left(\boldsymbol{S}_{0}^{T} \boldsymbol{S}_{0}+\boldsymbol{S}_{n}-\boldsymbol{S}_{0}^{T} \boldsymbol{\Gamma}_{\xi} \boldsymbol{S}_{T}\right) \boldsymbol{\Xi}= \\
& \boldsymbol{S}_{0}^{T}\left(\boldsymbol{A}_{\xi} \boldsymbol{S}_{0}+\boldsymbol{B}_{\xi} \boldsymbol{S}_{T}\right) \boldsymbol{V}+\boldsymbol{s}_{f} \boldsymbol{\xi}_{f}^{T} .
\end{aligned}
$$

We observe that $\boldsymbol{S}_{0}^{T} \boldsymbol{S}_{0}+\boldsymbol{S}_{n}=\boldsymbol{I}$, and $\boldsymbol{I}-\boldsymbol{S}_{0}^{T} \boldsymbol{\Gamma}_{\xi} \boldsymbol{S}_{T}$, being a triangular matrix with nonzero elements on the diagonal, is invertible. Finally, we find the explicit solution for the DCM waypoint matrix as

$$
\boldsymbol{\Xi}=\underbrace{\left[{ }^{\Xi} \boldsymbol{C}_{V}{ }^{\Xi} \boldsymbol{c}_{\xi}\right]}_{\Xi \boldsymbol{C}}\left[\begin{array}{c}
\boldsymbol{V} \\
\boldsymbol{\xi}_{f}^{T}
\end{array}\right]
$$

where

$$
\begin{aligned}
{ }^{\Xi} \boldsymbol{C}_{V} & =\left(\boldsymbol{I}-\boldsymbol{S}_{0}^{T} \boldsymbol{\Gamma}_{\xi} \boldsymbol{S}_{T}\right)^{-1} \boldsymbol{S}_{0}^{T}\left(\boldsymbol{A}_{\xi} \boldsymbol{S}_{0}+\boldsymbol{B}_{\xi} \boldsymbol{S}_{T}\right) \\
{ }^{\Xi} \boldsymbol{c}_{\xi} & =\left(\boldsymbol{I}-\boldsymbol{S}_{0}^{T} \boldsymbol{\Gamma}_{\xi} \boldsymbol{S}_{T}\right)^{-1} \boldsymbol{s}_{f} .
\end{aligned}
$$

CoM Waypoints: We start by evaluating (17) for $t=T_{\varphi}$ :

$\boldsymbol{x}_{\varphi, T}=\alpha_{\varphi, x_{T}} \boldsymbol{v}_{\varphi, 0}+\beta_{\varphi, x_{T}} \boldsymbol{v}_{\varphi, T}+\gamma_{\varphi, x_{T}} \boldsymbol{\xi}_{\varphi, T}+\delta_{\varphi, x_{T}} \boldsymbol{x}_{\varphi, 0}$,

where we used a similar notation for the coefficients as in (20), i.e., $\alpha_{\varphi, x_{T}}:=\alpha_{\varphi, x}\left(T_{\varphi}\right)$, etc. We write (25) in matrix form for all $n_{\varphi}$ phases as

$$
\boldsymbol{X}_{T}=\boldsymbol{A}_{x} \boldsymbol{V}_{0}+\boldsymbol{B}_{x} \boldsymbol{V}_{T}+\boldsymbol{\Gamma}_{x} \boldsymbol{\Xi}_{T}+\boldsymbol{\Delta}_{x} \boldsymbol{X}_{0},
$$

where $\boldsymbol{A}_{x}, \boldsymbol{B}_{x}, \boldsymbol{\Gamma}_{x}$, and $\boldsymbol{\Delta}_{x}$ are square, diagonal matrices containing the coefficients $\alpha_{\varphi, x_{T}}, \beta_{\varphi, x_{T}}, \gamma_{\varphi, x_{T}}$, and $\delta_{\varphi, x_{T}}$, respectively. The initial constraint for the CoM at the start of the motion, $\boldsymbol{x}_{1}=\boldsymbol{x}_{s}$, can be expressed in terms of $\boldsymbol{X}$ as

$$
\underbrace{\left[\begin{array}{cc}
1 & \mathbf{0}_{1 \times n_{\varphi}} \\
\mathbf{0}_{n_{\varphi} \times 1} & \mathbf{0}_{n_{\varphi} \times n_{\varphi}}
\end{array}\right]}_{\boldsymbol{S}_{1} \in \mathbb{R}^{n \times n}} \boldsymbol{X}=\underbrace{\left[\begin{array}{c}
1 \\
\mathbf{0}_{n_{\varphi} \times 1}
\end{array}\right]}_{\boldsymbol{s}_{s} \in \mathbb{R}^{n}} \boldsymbol{x}_{s}^{T} .
$$

Rewriting (26) in terms of $\boldsymbol{V}, \boldsymbol{\Xi}$, and $\boldsymbol{X}$, multiplying it on the left with $\boldsymbol{S}_{T}^{T}$, expanding $\boldsymbol{\Xi}$ according to (24), and adding (27), we find the explicit solution for the CoM waypoint matrix as

$$
\boldsymbol{X}=\underbrace{\left[\begin{array}{lll}
{ }^{X} \boldsymbol{C}_{V} & { }^{X} \boldsymbol{c}_{\xi} & { }^{X} \boldsymbol{c}_{x}
\end{array}\right]}_{{ }^{X} \boldsymbol{C}}\left[\begin{array}{c}
\boldsymbol{V} \\
\boldsymbol{\xi}_{f}^{T} \\
\boldsymbol{x}_{s}^{T}
\end{array}\right]
$$

where

$$
\begin{aligned}
{ }^{X} \boldsymbol{C}_{V} & =\left(\boldsymbol{I}-\boldsymbol{S}_{T}^{T} \boldsymbol{\Delta}_{x} \boldsymbol{S}_{0}\right)^{-1} \boldsymbol{S}_{T}^{T}\left(\boldsymbol{A}_{x} \boldsymbol{S}_{0}+\boldsymbol{B}_{x} \boldsymbol{S}_{T}+\boldsymbol{\Gamma}_{x} \boldsymbol{S}_{T}{ }^{\Xi} \boldsymbol{C}_{V}\right) \\
{ }^{X} \boldsymbol{c}_{\xi} & =\left(\boldsymbol{I}-\boldsymbol{S}_{T}^{T} \boldsymbol{\Delta}_{x} \boldsymbol{S}_{0}\right)^{-1} \boldsymbol{S}_{T}^{T} \boldsymbol{\Gamma}_{x} \boldsymbol{S}_{T}{ }^{\Xi} \boldsymbol{c}_{\xi} \\
{ }^{X} \boldsymbol{c}_{x} & =\left(\boldsymbol{I}-\boldsymbol{S}_{T}^{T} \boldsymbol{\Delta}_{x} \boldsymbol{S}_{0}\right)^{-1} \boldsymbol{s}_{s},
\end{aligned}
$$

${ }^{1}$ note that in our previous work [17], we used $\boldsymbol{\xi}_{n}=\boldsymbol{v}_{n}$ (the DCM comes to a stop at the end of the motion). Here, we use a more general constraint. 


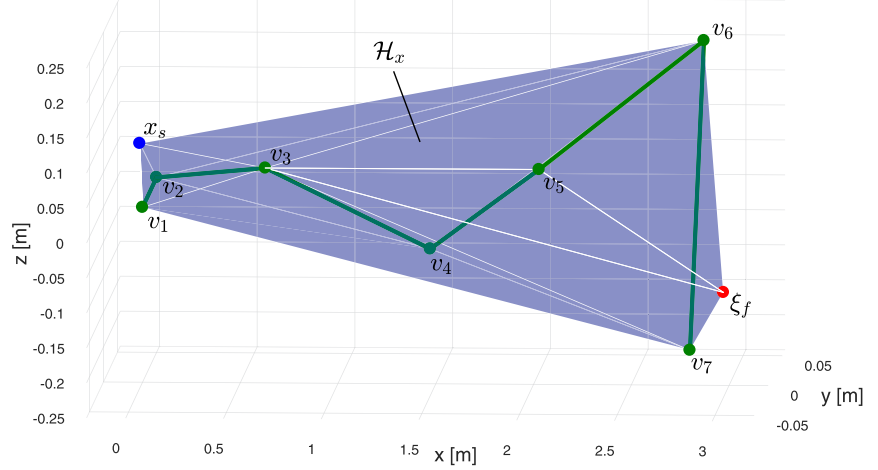

Fig. 5. CoM trajectory convex hull example.

and we used the identity $\boldsymbol{S}_{T}^{T} \boldsymbol{S}_{T}+\boldsymbol{S}_{1}=\boldsymbol{I}$. Note that $\boldsymbol{I}-\boldsymbol{S}_{T}^{T} \boldsymbol{\Delta}_{x} \boldsymbol{S}_{0}$ is a triangular matrix and is always invertible.

It can be verified that each row in ${ }^{X} \boldsymbol{C}$ consists of convex coefficients ${ }^{2}$ by expanding the compact matrix notation in terms of the transition phase coefficients, and using their respective convex properties. We can thus write for each CoM waypoint

$$
\boldsymbol{x}_{i} \in \mathcal{H}_{x}=\operatorname{CONv}\left(\left\{\boldsymbol{v}_{1}, \ldots, \boldsymbol{v}_{n}, \boldsymbol{\xi}_{f}, \boldsymbol{x}_{s}\right\}\right) .
$$

Figure 5 shows the CoM trajectory convex hull $\mathcal{H}_{x}$ for a trajectory consisting of 7 waypoints.

\section{WAYPOINT CONVEX Hulls}

In the previous section, we have treated the transition phase durations $T_{\varphi}$ as known, constant values. From a motion planning perspective, however, the phase durations are an output of the planning algorithm, and, therefore, not known a priori. In this section, we treat the durations as parameters, and we also reduce the domain of $T_{\varphi}$ to a closed interval $\mathbb{T}=[\underline{T}, \bar{T}] \subset \mathbb{R}_{>0}$, with the mention that we can choose $\underline{T}$ arbitrarily small and $\bar{T}$ arbitrarily large. ${ }^{3}$ Let $\boldsymbol{\tau}=\left[T_{1} \cdots T_{n_{\varphi}}\right]^{T}$ denote the vector of transition phase durations for the complete motion. The goal of the motion planner is to find the phase durations $\tau \in \mathbb{T}^{n_{\varphi}}$ that lead to feasible CoM trajectories with respect to kinematic and dynamic constraints. Ideally, the planner can treat each transition phase independently, enabling thus the usage of efficient binary search algorithms on the duration interval $\mathbb{T}$, as proposed in [16], instead of searching in the complete duration space $\mathbb{T}^{n_{\varphi}}$. However, changing the duration of one transition phase leads to new positions for all CoM waypoints, requiring recomputation and validation of the complete trajectory; the relation between phase durations and $\mathrm{CoM}$ waypoints is made explicit by rewriting (28) as

$$
\boldsymbol{X}(\boldsymbol{\tau})={ }^{X} \boldsymbol{C}(\boldsymbol{\tau})\left[\begin{array}{c}
\boldsymbol{V} \\
\boldsymbol{\xi}_{f}^{T} \\
\boldsymbol{x}_{s}^{T}
\end{array}\right] .
$$

\footnotetext{
${ }^{2}$ convex coefficients are nonnegative and sum to 1

${ }^{3}$ In practice, we use $\underline{T}=0.1 \mathrm{~s}$ and $\bar{T}=10 \mathrm{~s}$, as durations shorter than $0.1 \mathrm{~s}$ are usually associated with unfeasible forces and torques, while durations longer than $10 \mathrm{~s}$ are generally not desirable.
}

An alternative approach, circumventing this problem, is to use the locus of a CoM waypoint

$$
\mathcal{X}_{i}=\left\{\boldsymbol{x}_{i}(\boldsymbol{\tau}) \mid \boldsymbol{\tau} \in \mathbb{T}^{n_{\varphi}}\right\}
$$

as the set of possible CoM start points for the $i$-th transition phase. Then, multiple transition phase planners can run in parallel and find feasible pairs of CoM waypoints and phase durations, while a global planner coordinates the local planners and combines the transition phase trajectories into a complete feasible motion.

As an exact computation of the shape of $\mathcal{X}_{i}$ is, in general, not tractable, we propose in this work to generate a convex overapproximation by taking advantage of the convex properties presented in the previous sections. In (29), we obtained $\mathcal{H}_{x}$ (see Fig. 5 for an example), however this is a highly conservative overapproximation, as it uses all waypoints $\boldsymbol{v}_{i}$, and boundary points $\boldsymbol{\xi}_{f}$ and $\boldsymbol{x}_{s}$. We are interested in finding a tighter convex hull $\mathcal{H}_{x_{i}}$ for the CoM waypoint $\boldsymbol{x}_{i}$, with the property $\mathcal{X}_{i} \subset \mathcal{H}_{x_{i}} \subset \mathcal{H}_{x}$. The key insight is that the convex coefficients in ${ }^{X} C$ have lower and upper bounds, which depend only on our choice of the VRP interpolation function $f_{\varphi}$, and the transition phase duration bounds $\underline{T}$ and $\bar{T}$. Moreover, as $f_{\varphi}, \underline{T}$, and $\bar{T}$ are known a priori, the bounds of the ${ }^{X} \boldsymbol{C}$ coefficients can be precomputed. Using this information, we can find a new set of points that define the reduced convex hull $\mathcal{H}_{x_{i}}$ with the following algorithm.

Convex hull with bounded coefficients (Algorithm 1): Let $\mathcal{P}=\left\{\boldsymbol{p}_{i}\right\}_{i=1}^{N}$ be a set of $N$ three-dimensional points, for which we define a convex hull with bounded coefficients

$$
\mathcal{P}_{A}=\left\{\boldsymbol{p} \mid \boldsymbol{p}=\sum_{i=1}^{N} a_{i} \boldsymbol{p}_{i}, \sum_{i=1}^{N} a_{i}=1, a_{i} \in\left[\underline{a}_{i}, \bar{a}_{i}\right]\right\},
$$

where each coefficient $a_{i}$ has nonnegative lower and upper bounds, $\underline{a}_{i}$ and $\bar{a}_{i}$, respectively. Then, our goal is to find the vertices of $\mathcal{P}_{A}$, or, in more general terms, a set of $M$ points $\mathcal{Q}=\left\{\boldsymbol{q}_{j}\right\}_{j=1}^{M}$ such that $\operatorname{CoNV}(\mathcal{Q})=\mathcal{P}_{A}$; note that $\operatorname{VerTICES}\left(\mathcal{P}_{A}\right) \subseteq \mathcal{Q}$. The main idea of the algorithm is to rephrase the definition of $\mathcal{P}_{A}$ : instead of a set of $N$ threedimensional points multiplied by convex coefficients, we can describe $\mathcal{P}_{A}$ as a set of $N$-dimensional points $\boldsymbol{a}=\left[a_{1} \cdots a_{N}\right]^{T}$ projected to the three-dimensional space by multiplication with a matrix $\boldsymbol{P}=\left[\boldsymbol{p}_{1} \cdots \boldsymbol{p}_{N}\right]$. Finding the vertices of $\mathcal{P}_{A}$ is thus equivalent to finding the vertices of the $\mathrm{N}$-dimensional shape corresponding to the constraints $\sum_{i=1}^{N} a_{i}=1$ and $a_{i} \in\left[\underline{a}_{i}, \bar{a}_{i}\right]$.

We start by creating an $N$-dimensional hyperrectangle $\mathcal{O}_{R}$ as the Cartesian product of the closed intervals $\left[\underline{a}_{i}, \bar{a}_{i}\right]$ (line 1.2, i.e., Algorithm 1, line 2), and the hyperplane $\mathcal{A}$ with equation $\sum_{i=1}^{N} a_{i}=1$, corresponding to the convexity condition (line 1.3). The intersection $\mathcal{O}_{A}=\mathcal{O}_{R} \cap \mathcal{A}$ is an $N$-dimensional hyperpolygon with $M$ vertices $\mathcal{O}=\left\{\boldsymbol{o}_{j}\right\}_{j=1}^{M}$ (line 1.4). Projecting the vertices $\mathcal{O}$ to the three-dimensional space yields $\mathcal{Q}$ (line 1.5), and $\operatorname{Conv}(\mathcal{Q})=\operatorname{Conv}(\boldsymbol{P} \mathcal{O})=\boldsymbol{P} \operatorname{Conv}(\mathcal{O})=\boldsymbol{P} \mathcal{O}_{A}=\mathcal{P}_{A}$ proves that we found the appropriate set $\mathcal{Q}$.

Figure 6 shows a two-dimensional example with three points forming a triangle $(N=3)$, and the bounded convex hull as a polygon with five vertices $(M=5)$. In this particular example, all points in $\mathcal{Q}$ are vertices of $\mathcal{P}_{A}$. 

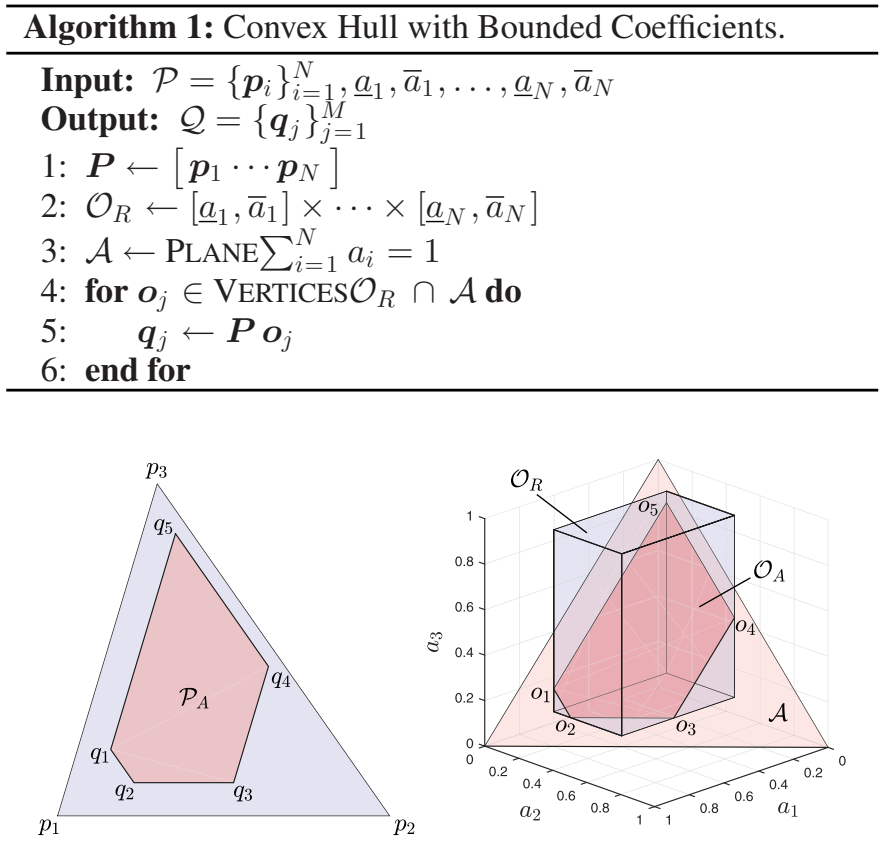

Fig. 6. Bounded convex hull example.

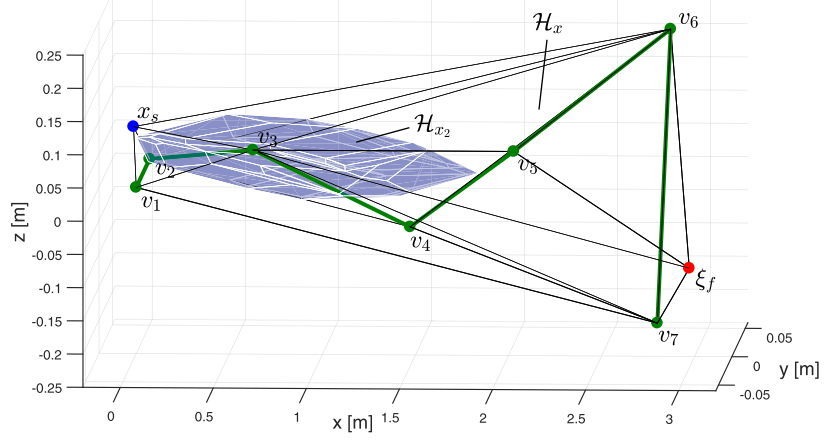

Fig. 7. CoM waypoint bounded convex hull example.

Using the algorithm presented above, we computed the bounded convex hulls $\mathcal{H}_{x_{i}}$ for the example in Fig. 5, and obtained significantly reduced convex hulls. Figure 7 shows the bounded convex hull $\mathcal{H}_{x_{2}}$ of the second CoM waypoint $\boldsymbol{x}_{2}$ from our multi-step example (the first waypoint is the CoM reference start point $\boldsymbol{x}_{s}$, so $\mathcal{H}_{x_{1}}=\left\{\boldsymbol{x}_{s}\right\}$ ). The volume of $\mathcal{H}_{x_{2}}$ is $16.8 \%$ of the volume of the trajectory convex hull $\mathcal{H}_{x}$, computed previously.

\section{OPTIMAL VRP PlaCEMENT USING COM WAYPOINT CONVEX HULLS}

In our previous work [16], we presented a multi-contact transition planner for humanoid robots, which uses a sequence of manually chosen VRP waypoints to generate feasible CoM trajectories. Automatic VRP placement has been the focus of our recent research with the goal of increasing the autonomy of the robot. In this work, we present preliminary results of using the CoM waypoint convex hulls for optimal VRP placement.

Before we proceed, we recapitulate several definitions from [16]: a contact is a tuple $c=\left(\boldsymbol{p}_{c}, \boldsymbol{R}_{c}\right)$, where $\boldsymbol{p}_{c}$ is the three-dimensional point of contact, and $\boldsymbol{R}_{c} \in S O(3)$ is the ori- entation of the contact frame, chosen such that the $z$-axis is aligned with the contact surface normal, a stance [20] is a set of $K$ contacts $\sigma=\left\{c_{k}\right\}_{k=1}^{K}$, and a contact wrench is a generalized force on $S E(3)$ written as a six-dimensional vector $\boldsymbol{w}_{c}=\left[\begin{array}{ll}\boldsymbol{f}_{c}^{T} & \boldsymbol{\tau}_{c}^{T}\end{array}\right]^{T}$ [21], where $\boldsymbol{f}_{c}$ denotes the force, and $\boldsymbol{\tau}_{c}$ is the torque. The contact wrench acts at the contact point $\boldsymbol{p}_{c}$ and is expressed in the contact frame $\boldsymbol{R}_{c}$. Given a stance $\sigma$ and the set of contact wrenches $\boldsymbol{w}_{c_{k}}$, we can compute the total wrench acting on the CoM as

$$
\boldsymbol{w}_{x}=\sum_{k=1}^{K} \underbrace{\left[\begin{array}{cc}
\boldsymbol{R}_{c_{k}} & \mathbf{0}_{3 \times 3} \\
{\left[\left(\boldsymbol{p}_{c_{k}}-\boldsymbol{x}\right)^{\circ} \times \boldsymbol{R}_{c_{k}}\right.} & \boldsymbol{R}_{c_{k}}
\end{array}\right]}_{\boldsymbol{G}_{k}(\boldsymbol{x})} \boldsymbol{w}_{c_{k}}=\boldsymbol{G}_{\sigma}(\boldsymbol{x}) \boldsymbol{w}_{\sigma},
$$

where $\boldsymbol{G}_{\sigma}(\boldsymbol{x})=\left[\boldsymbol{G}_{1}(\boldsymbol{x}) \cdots \boldsymbol{G}_{K}(\boldsymbol{x})\right]$, and the contact wrenches were stacked into $\boldsymbol{w}_{\sigma}=\left[\boldsymbol{w}_{c_{1}}^{T} \cdots \boldsymbol{w}_{c_{K}}^{T}\right]^{T}$. Conversely, given a desired CoM wrench $\boldsymbol{w}_{x}^{d}$ and a CoM position $\boldsymbol{x}$, we can find the necessary contact wrenches $\boldsymbol{w}_{\sigma}^{d}(\boldsymbol{x})$ fulfilling dynamic constraints (contact unilaterality, friction cone constraints, bounded normal force, and center of pressure constraints), by solving a constrained quadratic optimization problem

$$
\boldsymbol{w}_{\sigma}^{d}(\boldsymbol{x})=\underset{\boldsymbol{w}}{\operatorname{argmin}}\left\|\boldsymbol{G}_{\sigma}(\boldsymbol{x}) \boldsymbol{w}-\boldsymbol{w}_{x}^{d}\right\|_{\boldsymbol{Q}_{w}}^{2},
$$

where $\boldsymbol{Q}_{w}$ is a symmetric, positive definite weighting matrix. The CoM position $\boldsymbol{x}$ is dynamically feasible for the stance $\sigma$ and the desired wrench $\boldsymbol{w}_{x}^{d}$ if the magnitude of the residual wrench vector

$$
\varepsilon_{\sigma}^{d}(\boldsymbol{x})=\boldsymbol{G}_{\sigma}(\boldsymbol{x}) \boldsymbol{w}_{\sigma}^{d}(\boldsymbol{x})-\boldsymbol{w}_{x}^{d}
$$

is less than a tolerance value $\varepsilon$. Further, it is kinematically feasible for the stance $\sigma$ if the distances between the CoM position $\boldsymbol{x}$ and the contact points $\boldsymbol{p}_{c_{k}}$ are within lower and upper bounds determined by the robot kinematic constraints [22]. Finally, we define the function $\operatorname{FeAsible}\left(\boldsymbol{x}, \sigma, \boldsymbol{w}_{x}^{d}\right)$ to return true if the CoM position $\boldsymbol{x}$ is kinematically and dynamically feasible for the stance $\sigma$ and the desired wrench $\boldsymbol{w}_{x}^{d}$, and false otherwise.

Optimal VRP placement algorithm: Given a sequence of stances $\left(\sigma_{i}\right)_{i=1}^{n}$, we search for an optimal sequence of $n$ VRP waypoints $\left(\boldsymbol{v}_{i}\right)_{i=1}^{n}$. Choosing one VRP waypoint for each stance is motivated by generalizing a walking motion with alternating single support and double support phases to multi-contact locomotion. In this work, we find optimal VRP waypoints using a two-step process.

First, for each stance $\sigma_{i}$, we search for a VRP waypoint $\boldsymbol{v}_{i}$ that can act as CoM rest position, i.e., $\boldsymbol{v}_{i}$ is a feasible CoM position

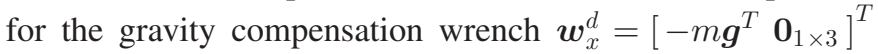
and stance $\sigma_{i}$. Here, $\boldsymbol{g}=\left[\begin{array}{lll}0 & 0 & -g\end{array}\right]^{T}$ denotes the gravitational acceleration vector. We find $\boldsymbol{v}_{i}$ by solving the optimization problem

$$
\boldsymbol{v}_{i}=\underset{\boldsymbol{x}}{\operatorname{argmin}}\left(\left\|\boldsymbol{\varepsilon}_{\sigma_{i}}^{d}(\boldsymbol{x})\right\|_{\boldsymbol{Q}_{d}}^{2}+\left\|\boldsymbol{w}_{\sigma_{i}}^{d}(\boldsymbol{x})\right\|_{\boldsymbol{Q}_{\sigma}}^{2}+\left\|\boldsymbol{x}_{z}-\Delta z\right\|_{\boldsymbol{Q}_{z}}^{2}\right),
$$

where $\boldsymbol{Q}_{d}, \boldsymbol{Q}_{\sigma}$, and $\boldsymbol{Q}_{z}$ are symmetric, positive definite weighting matrices, $\boldsymbol{x}_{z}$ is the vertical component of $\boldsymbol{x}$, and $\Delta z$ was introduced in Section III. The first term in (36) attempts to place $\boldsymbol{v}_{i}$ within the region of static equilibrium, the second term reduces the required contact torques and tangential forces for static 
equilibrium, while the third term ensures that the VRP waypoint is consistent with the definition of the time constant $b$. We use the VRP waypoints $\left(\boldsymbol{v}_{i}\right)_{i=1}^{n}$ as an initial sequence of waypoints for the nonlinear optimization process in the subsequent steps.

Second, we use the feasibility of CoM waypoints sampled from the convex hulls $\mathcal{H}_{x_{i}}$ to define a cost function for an arbitrary VRP waypoint sequence. As discussed in the previous section, a convex hull $\mathcal{H}_{x_{i}}$ is an overapproximation of the CoM waypoint locus $\mathcal{X}_{i}(31)$ with respect to the durations of all transition phases. Although the transition phase durations and the timing of the stance transitions are not known (they are computed by the motion planner from [16] given a sequence of VRP waypoints), we can state that during the stance $\sigma_{i}$, the CoM trajectory will pass through a waypoint within $\mathcal{X}_{i}$. This is a direct consequence of associating the VRP waypoint $\boldsymbol{v}_{i}$ with the stance $\sigma_{i}$. This insight motives the introduction of a VRP cost function based on the feasibility of CoM positions within $\mathcal{H}_{x_{i}}$ with respect to the stance $\sigma_{i}$. Given an arbitrary VRP waypoint sequence $\left(\boldsymbol{v}_{i}\right)_{i=1}^{n}$, we construct the matrix $\boldsymbol{V}$, take $\boldsymbol{x}_{s}=\boldsymbol{v}_{1}$ and $\boldsymbol{\xi}_{f}=\boldsymbol{v}_{n}$, and compute the bounded convex hull $\mathcal{H}_{x_{i}}$ as the finite set of points $\mathcal{Q}_{x_{i}}(\boldsymbol{V})$ using Algorithm $1\left(\mathcal{H}_{x_{i}}=\operatorname{CONV}\left(\mathcal{Q}_{x_{i}}\right)\right)$. For each point $\boldsymbol{q}_{x_{i}} \in \mathcal{Q}_{x_{i}}(\boldsymbol{V})$, we compute the wrench $\boldsymbol{w}_{x_{i}}=\left[\boldsymbol{f}_{x_{i}}^{T} \mathbf{0}_{1 \times 3}\right]^{T}$, with $\boldsymbol{f}_{x_{i}}=\frac{m}{b^{2}}\left(\boldsymbol{q}_{x_{i}}-\boldsymbol{v}_{i}\right)-m \boldsymbol{g}$, according to the definitions given in Section II. Let $\mathcal{F}_{x_{i}}$ be the set of feasible points in $\mathcal{Q}_{x_{i}}$ under the feasibility definition introduced above:

$$
\mathcal{F}_{x_{i}}(\boldsymbol{V})=\left\{\boldsymbol{q}_{x_{i}} \in \mathcal{Q}_{x_{i}}(\boldsymbol{V}) \mid \operatorname{FEASIBLE}\left(\boldsymbol{q}_{x_{i}}, \sigma_{i}, \boldsymbol{w}_{x_{i}}\right)\right\} .
$$

We define the cost function for $\boldsymbol{V}$ using the cardinality of the feasible set $\mathcal{F}_{x_{i}}(\boldsymbol{V})$ :

$$
g(\boldsymbol{V})=\sum_{i=1}^{n}\left(1-\frac{\# \mathcal{F}_{x_{i}}(\boldsymbol{V})}{\# \mathcal{Q}_{x_{i}}(\boldsymbol{V})}\right)
$$

We also considered sampling uniformly a set of points from $\mathcal{H}_{x_{i}}$, which better approximates the shape of the convex hull. However, this method incurs a performance penalty, as we need to compute explicitly the convex hulls $\mathcal{H}_{x_{i}}$ for each evaluation of the cost function. Here, we use the points $\mathcal{Q}_{x_{i}}$ for performance reasons, as they can be computed linearly from $\boldsymbol{V}$.

Finally, we obtain optimal VRP waypoints via nonlinear optimization

$$
\boldsymbol{V}_{\text {opt }}=\underset{\boldsymbol{V}}{\operatorname{argmin}} g(\boldsymbol{V}) \text {. }
$$

Intuitively, the optimization process increases the space of feasible durations, thus aiding the motion planner in finding a feasible CoM trajectory. Note that each iteration of the optimization process requires multiple evaluations of the function $\operatorname{FEASIBLE}\left(\boldsymbol{q}_{x_{i}}, \sigma_{i}, \boldsymbol{w}_{x_{i}}\right)$, which in turn requires solving the constrained quadratic optimization (34). Alternatively, for our future work, we consider generating a convex hull of feasible wrenches $\mathcal{H}_{w}$ given a stance $\sigma_{i}$, and verifying the dynamic feasibility of a wrench $\boldsymbol{w}_{x_{i}}$ by checking whether $\boldsymbol{w}_{x_{i}} \in \mathcal{H}_{w}$.

\section{IMPLEMENTATION DETAILS AND SIMULATION RESULTS}

To validate the presented approach, we implemented the described algorithms in Matlab, and executed a multi-contact scenario in simulation (Fig. 8), in which the humanoid robot

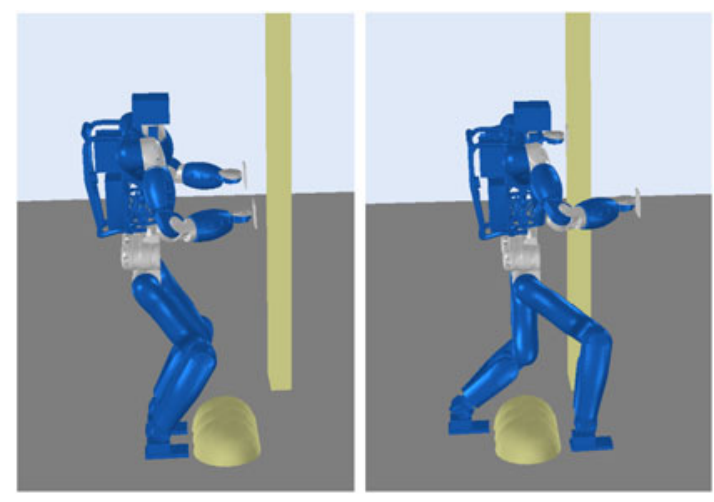

Fig. 8. Multi-contact scenario (initial and final stance).

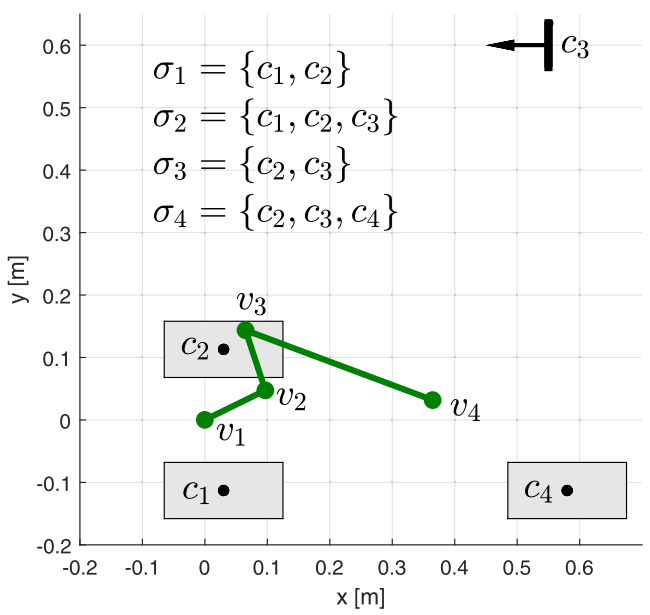

Fig. 9. Multi-contact transition example.

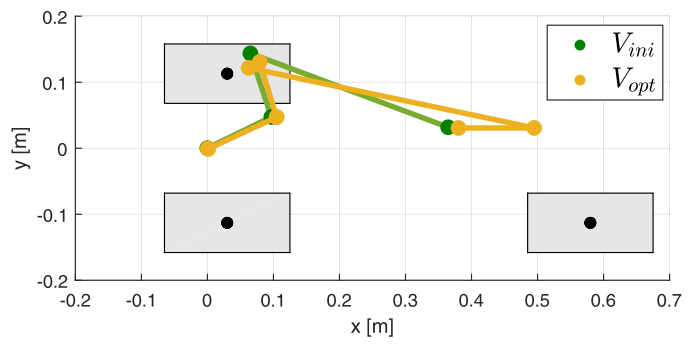

Fig. 10. Optimal VRP placement example.

TORO [23] uses its left hand for support while stepping with the right foot over a large hemispherical obstacle. Figure 9 shows the contacts $c_{1}$ and $c_{4}$ for the right foot, $c_{2}$ for the left foot, and $c_{3}$ for the left hand, each with their respective normals, as well as the sequence of stances $\left(\sigma_{i}\right)_{i=1}^{4}$. We found the initial sequence of VRP waypoints $\left(\boldsymbol{v}_{i}\right)_{i=1}^{4}$ (Fig. 9) within 0.43 seconds by solving (36) for each stance with the built-in Matlab optimization solver fminsearch.

We create the VRP sequence $\boldsymbol{V}_{\text {in } i}$ by adding each VRP twice $\boldsymbol{V}_{\text {ini }}=\left[\begin{array}{llllllll}\boldsymbol{v}_{1} & \boldsymbol{v}_{1} & \boldsymbol{v}_{2} & \boldsymbol{v}_{2} & \boldsymbol{v}_{3} & \boldsymbol{v}_{3} & \boldsymbol{v}_{4} & \boldsymbol{v}_{4}\end{array}\right]^{T}$, according to the requirements of the multi-contact transition planner from [16]. We use $\boldsymbol{V}_{\text {ini } i}$ as initial guess to obtain optimal VRP waypoints $\boldsymbol{V}_{\text {opt }}$. We solved (39) using fminsearch, which found a solution (Fig. 10) in 27.8 seconds after 200 iterations, reducing the cost from $g\left(\boldsymbol{V}_{\text {ini }}\right)=0.5$ to $g\left(\boldsymbol{V}_{\text {opt }}\right)=0.42$. 


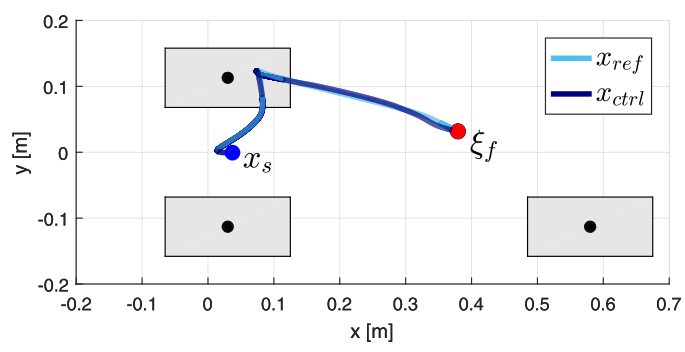

Fig. 11. CoM reference trajectory tracking example.

Subsequently, the multi-contact transition planner from [16] found the phase durations $\left(T_{\varphi}\right)_{\varphi=1}^{7}$ and stance transition times for a feasible motion plan with a total duration of 3.8 seconds; the planner execution time was 1.7 seconds. The reference $\mathrm{CoM}$ trajectory was tracked successfully (Fig. 11) by the passivitybased whole-body controller introduced by Henze et al. [24], running in simulation with OpenHRP [25].

\section{CONCLUSION AND FUTURE WORK}

In this letter, we have formally proven the convex properties of the DCM and CoM trajectories, and discussed them in the context of trajectories generated using a piecewise interpolation approach over a sequence of VRP waypoints. Further, we have introduced an algorithm for computing the convex hull of a set of points with bounded convex coefficients, and we used it to obtain convex overapproximations for the CoM waypoints. As an application example in the context of multicontact locomotion planning, the CoM waypoint convex hulls can be used to find optimal VRP waypoints given a sequence of stances (chosen manually or obtained from a contact planner). We have discussed the current limitations of the algorithm and intended approaches to circumvent them in the corresponding section.

As future work, we plan to combine our DCM-based approach and convex approximations of the CoM waypoints with the complementary concept of the CoM convex stability region [13]. Additionally, we will work towards the goal of creating a fast, autonomous motion planning algorithm, which can interact closely with the whole-body controller to achieve robust locomotion in challenging environments.

\section{REFERENCES}

[1] S. Lengagne, J. Vaillant, E. Yoshida, and A. Kheddar, "Generation of whole-body optimal dynamic multi-contact motions," Int. J. Robot. Res., vol. 32, no. 9-10, pp. 1104-1119, 2013.

[2] L. Saab, O. E. Ramos, F. Keith, N. Mansard, P. Soueres, and J.-Y. Fourquet, "Dynamic whole-body motion generation under rigid contacts and other unilateral constraints," IEEE Trans. Robot., vol. 29, no. 2, pp. 346-362, Apr. 2013.

[3] R. J. Full and D. E. Koditschek, "Templates and anchors: Neuromechanical hypotheses of legged locomotion on land," J. Exp. Biol., vol. 202, no. 23, pp. 3325-3332, 1999.
[4] M. Vukobratović and J. Stepanenko, "On the stability of anthropomorphic systems," Math. Biosci., vol. 15, no. 1-2, pp. 1-37, 1972.

[5] S. Kajita, F. Kanehiro, K. Kaneko, K. Yokoi, and H. Hirukawa, "The 3D linear inverted pendulum mode: A simple modeling for a biped walking pattern generation," in Proc. IEEE/RSJ Int. Conf. Intell. Robots Syst., vol. 1, 2001, pp. 239-246.

[6] K. Harada, S. Kajita, K. Kaneko, and H. Hirukawa, "An analytical method for real-time gait planning for a humanoid robot," Int. J. Humanoid Robot., vol. 3, no. 1, pp. 1-19, 2006.

[7] S. Kajita et al., "Biped walking pattern generation by using preview control of zero-moment point," in Proc. IEEE Int. Conf. Robot. Automat., vol. 2, 2003, pp. 1620-1626.

[8] R. Tedrake, S. Kuindersma, R. Deits, and K. Miura, "A closed-form solution for real-time ZMP gait generation and feedback stabilization," in Proc. IEEE-RAS 15th Int. Conf. Humanoid Robots, 2015, pp. 936-940.

[9] H. Hirukawa et al., "A universal stability criterion of the foot contact of legged robots-Adios ZMP," in Proc. IEEE Int. Conf. Robot. Automat., 2006, pp. 1976-1983.

[10] S. Caron, Q.-C. Pham, and Y. Nakamura, "Leveraging cone double description for multi-contact stability of humanoids with applications to statics and dynamics," in Proc. Robotics: Science and Systems, Rome, Italy, Jul. 2015, doi: 10.15607/RSS.2015.XI.028.

[11] H. Dai and R. Tedrake, "Planning robust walking motion on uneven terrain via convex optimization," in Proc. IEEE-RAS 16th Int. Conf. Humanoid Robots, 2016, pp. 579-586.

[12] T. Bretl and S. Lall, "Testing static equilibrium for legged robots," IEEE Trans. Robot., vol. 24, no. 4, pp. 794-807, Aug. 2008.

[13] H. Audren and A. Kheddar, "3-D robust stability polyhedron in multicontact," IEEE Trans. Robot., vol. 34, no. 2, pp. 388-403, Apr. 2018.

[14] J. Englsberger, C. Ott, and A. Albu-Schäffer, "Three-dimensional bipedal walking control using divergent component of motion," in IEEE/RSJ Int. Conf. Intell. Robots Syst., 2013, pp. 2600-2607.

[15] T. Koolen, T. De Boer, J. Rebula, A. Goswami, and J. Pratt, "Capturabilitybased analysis and control of legged locomotion, part 1: Theory and application to three simple gait models," Int. J. Robot. Res., vol. 31, no. 9, pp. 1094-1113, 2012.

[16] G. Mesesan, J. Englsberger, B. Henze, and C. Ott, "Dynamic multi-contact transitions for humanoid robots using divergent component of motion," in Proc. IEEE Int. Conf. Robot. Automat., 2017, pp. 4108-4115.

[17] J. Englsberger, G. Mesesan, and C. Ott, "Smooth trajectory generation and push-recovery based on divergent component of motion," in Proc. IEEE/RSJ Int. Conf. Intell. Robots Syst., 2017, pp. 4560-4567.

[18] P. Varaiya, "Reach set computation using optimal control," in Verification of Digital and Hybrid Systems. Berlin, Heidelberg, Germany: Springer, 2000, pp. 323-331.

[19] J. LeMay, "Recoverable and reachable zones for control systems with linear plants and bounded controller outputs," IEEE Trans. Autom. Control, vol. 9, no. 4, pp. 346-354, Oct. 1964.

[20] K. Bouyarmane and A. Kheddar, "Humanoid robot locomotion and manipulation step planning," Adv. Robot., vol. 26, no. 10, pp. 1099-1126, 2012.

[21] R. M. Murray, Z. Li, S. S. Sastry, and S. S. Sastry, A Mathematical Introduction to Robotic Manipulation. Boca Raton, FL, USA: CRC Press, 1994.

[22] O. Porges, T. Stouraitis, C. Borst, and M. A. Roa, "Reachability and capability analysis for manipulation tasks," in ROBOT2013: First Iberian Robotics Conference. Cham, Switzerland: Springer, 2014, pp. 703-718.

[23] J. Englsberger et al., "Overview of the torque-controlled humanoid robot TORO," in Proc. IEEE-RAS Int. Conf. Humanoid Robots, 2014, pp. 916-923.

[24] B. Henze, M. A. Roa, and C. Ott, "Passivity-based whole-body balancing for torque-controlled humanoid robots in multi-contact scenarios," Int. J. Robot. Res., vol. 35, no. 12, pp. 1522-1543, 2016.

[25] F. Kanehiro, H. Hirukawa, and S. Kajita, "OpenHRP: Open architecture humanoid robotics platform," Int. J. Robot. Res., vol. 23, no. 2, pp. 155-165, 2004. 